\title{
Prediction of Electromagnetic Fields around High Voltage Transmission Lines
}

\author{
G. A. Kulkarni ${ }^{1}$, W. Z. Gandhare ${ }^{2}$ \\ ${ }^{1}$ Research Scholar, Govt. College of Engineering, Dept of Electrical Engineering, \\ Osmanpur, 431005 Aurangabad, India \\ E-mail: girish227252@rediffmail.com \\ ${ }^{2}$ Principal, Marathwada Mitra Mandal's Institute of Technology, Lohgaon, Pune, \\ 411047, India \\ E-mail: wz_gandhare@yahoo.co.in
}

Abstract: The electric and magnetic fields are present around the High Voltage Transmisssion Lines (HVTL) and reported to affect health of the workers working on these hotlines. The key parameter reportedly responsible for detrimental health effects, are internal induced fields in body. Induction of internal fields in different organs is heavily dependent on the external fields. Prediction of hazardous levels of external fields before measurement or in situations difficult for direct measurement will lead to identify the restrictive situations and working conditions for hotline workers. This work propose a method to model electric and magnetic field for different climbing routes using hybrid technique, formed by combining support vector machine (SVM) and neural network (NN) and also electric field and magnetic field values are predicted using NN for increase in tower height. The result shows the performance of proposed method for prediction of electric field and magnetic field for increase in tower height.

Keywords: climbing routes, support vector machines, neural network, hybrid data mining approach

\section{Introduction}

Rapid expansion of power systems is causing transmission technology to drift from HV to EHV. This HVTL network is expanding with a great speed along and above the ground. Current and voltage limits are the two significant factors of high voltage transmission line [1]. Large transmission line configurations with high voltage and current levels produce large values of electric and magnetic fields stresses which influence the humans and nearby objects sited at ground surfaces. This has in turn prompted increased activity in the documentation of calculation methods to exactly forecast field strengths in isolated conducting bodies associated with lines of all voltages and design configurations [2]. Due to the extensive use of electricity in the modern domestic and industrial environment, any or all reports purporting to exhibit 
that the electric fields from power lines that cause or aggravate infirmity must be given serious consideration and be seriously evaluated [3].

The exposure of general public and power-line workers to high-voltage transmission lines at extremely low frequency (ELF) of 50 or $60 \mathrm{~Hz}$ may cause very severe health problems [4]. The human body is always vulnerable to electromagnetic radiation of varying intensity depending upon the locality.

Modeling of external fields was done using Regression analysis, SVM, NN and Hybrid Techniques [5]. In this paper a hybrid technique is used to model electric field and magnetic field present around hotline workers body while climbing through different climbing routes in a tower and also electric field and magnetic field was predicted as increase in the tower height.

\section{Modeling and Prediction using Hybrid Technique}

Extensive efforts carried out, to evaluate external exposure conditions and its effects reported in literature can be found out elsewhere [6, 7]. In almost all these attempts to determine the detrimental effects, electromagnetic fields are measured along the span of the line. Reducing distance between source and object, while doing live line repairs has also attracted attention of researchers to determine biological effects initiated by electromagnetic fields [6]. Prediction of fields during different positions of HVTL tower climbing routes will be helpful to avoid extreme exposure prone spots during live line maintenance.

Artificial neural networks, SVM along with classical statistical procedures such as polynomial regression are used for function approximation. These methods have succeeded in generating accurate plots, provided the input data is sufficient.

Recently, Hybrid data mining approaches have gained much popularity. A hybrid approach is built by combining two or more data mining techniques.

Reviewed literature indicates that Hybrid models can outperform standalone models and can provide better performance. The objective behind formation of Hybrid model is to extract all possible good features of individual model to obtain best possible outcomes. Generating new hybrid model by mixing individual models has also been suggested by Xu, Kryzak, and Suen (1992) [8].

Development of hybrid models by combining different NNs architectures were also reported and showed that the combinations provided improved performance compared to standalone NNs models. Hybrid models by combining statistical models (mixed regression models) have been implemented. Results from these studies suggest that hybrid models improve predictive performance when compared against the predictive performances of standalone models. The recent trend in hybrid model development is to mix classical statistical models with NNs models [9].

On modeling side, a need persists to develop a technique with conventional methods to model the external field values. There is a scope identified to implement a hybrid technique which can outperform the advantages of standalone techniques such as Neural Network and Support Vector Machines. Possibility of developing a hybrid technique using NN and SVM is exploited in this work for important issues of electromagnetic fields. 
Here a new method is proposed for modeling of the electric field and magnetic field in different climbing routes of a tower and also to predict the electric field and magnetic field, if height of the tower also increases. The modeling of electric field and magnetic field is performed by using various functions such as basic fitting function, support vector machine (SVM) and neural network. Prediction of electric field and magnetic field is done for increase in tower height.

\section{Methodology}

In proposed method curve fitting technique is used for the modeling of electromagnetic field for various climbing routes.

Curve fitting is a process of generating a curve which best represents the characteristics of a system using the input data set. It is the basis of any analytical, comparative or growth related statistics. The objective [10] of curve fitting is to select parameter values which minimize the total error over the set of data points being considered.

The error is calculated by observing the vertical distance between the line and the point $\left(x_{\mathrm{i}}, y_{\mathrm{i}}\right)$, which is given by [14]

$$
E_{i}=\left|y_{i}-m x_{i}-b\right|
$$

The idea behind the least squares method is to sum these vertical distances and minimize the total error. The least square error is

$$
E(m, b)=\sum_{k=1}^{n}\left(y_{k}-m x_{k}-b\right)^{2}
$$

In order to maximize or minimize a function of multiple variables, we compute the partial derivatives with respect to each of the variables and set them equal to zero. Here, we compute

$$
\begin{aligned}
& \frac{\partial E}{\partial m}=-2 \sum_{k=1}^{n} x_{k}\left(y_{k}-m x_{k}-b\right)=0 \\
& \frac{\partial E}{\partial b}=-2 \sum_{k=1}^{n}\left(y_{k}-m x_{k}-b\right)=0
\end{aligned}
$$

which can be solved as a linear system of two equations for the two unknowns $m$ and $b$. Since $m$ and $b$ are uniquely determined, these values yield the minimum error. Similarly we can proceed for any polynomial. For second degree polynomials of the form

$$
y=a_{0}+a_{1} x+a_{2} x^{2}
$$

the error becomes

$$
E\left(a_{0}, a_{1}, a_{2}\right)=\sum_{k=1}^{n}\left(y_{k}-a_{0}-a_{1} x_{k}-a_{2} x_{k}^{2}\right)^{2}
$$


Initially the electric field and magnetic field values for different tower height which are also different for climbing routes are taken and using this data the curve is plotted by using basic curve fitting function. The experimental dataset used to plot curve in the proposed method is shown in equation $7 \& 8$.

$$
\begin{gathered}
D_{E}=\left[\begin{array}{c}
\mathrm{H}_{1} \mathrm{E}_{1} \\
\mathrm{H}_{2} \mathrm{E}_{2} \\
\vdots \\
\mathrm{H}_{\mathrm{n}} \mathrm{E}_{\mathrm{n}}
\end{array}\right] \\
\mathrm{D}_{\mathrm{M}}=\left[\begin{array}{c}
\mathrm{H}_{1} \mathrm{M}_{1} \\
\mathrm{H}_{2} \mathrm{M}_{2} \\
\vdots \\
\mathrm{H}_{\mathrm{n}} \mathrm{M}_{\mathrm{n}}
\end{array}\right]
\end{gathered}
$$

The above dataset is given as an input data to the basic curve fitting function and then curve is plotted for linear, quadratic, cubic, $4^{\text {th }}$ degree polynomial, $5^{\text {th }}$ degree polynomial, $6^{\text {th }}$ degree polynomial, $7^{\text {th }}$ degree polynomial, $8^{\text {th }}$ degree polynomial, $9^{\text {th }}$ degree polynomial and $10^{\text {th }}$ degree polynomial. After plotting the curve, the best curve is taken as the experimental data curve for different climbing routes. The next step after selection of the best curve is to train SVM and neural network to predict for the electric field and magnetic field for increase in tower height.

\subsection{Prediction of Electric and Magnetic Field for Increase in Tower Height}

Artificial Neural Network (ANN) plays an important role while in prediction of the linear and non-linear problems in different fields of engineering [11]. Usually neural network consists of two stages namely; training stage and testing stage. In the first stage, neural network is trained by using the training dataset and in the second stage; it provides the predicted electric field and magnetic field to the corresponding height of the tower. For the training purposes of neural network back propagation algorithm is used.

SVMs are powerful machine learning techniques for classification and regression [12]. In proposed method SVM is used for prediction of the electric field and magnetic field for increase in tower height. SVM is classified into two different types binary classifier based SVM and multiclass classifier based SVM. Here more than two classes are used, so multiclass SVM classifier is used.

The hybrid technique presented is a combination of SVM and neural network. Here, the best data from SVM and neural network are taken and curve is plotted for electric field and magnetic field. For prediction, if height of the tower is given as the input; the hybrid technique gives output as corresponding electric and magnetic field.

\section{Result and discussions}

The implementation of proposed method was performed by using MATLAB 7.11 and the proposed method is tested for HVTL experimental data. The HVTL experimental data is taken from [13]. 


\subsection{Prediction and modeling for HVTL data}

The HVTL experiment data for electric field and magnetic field are considered for different climbing routes in a tower. Different climbing routes [13] considered in our method are shown in figure 1.

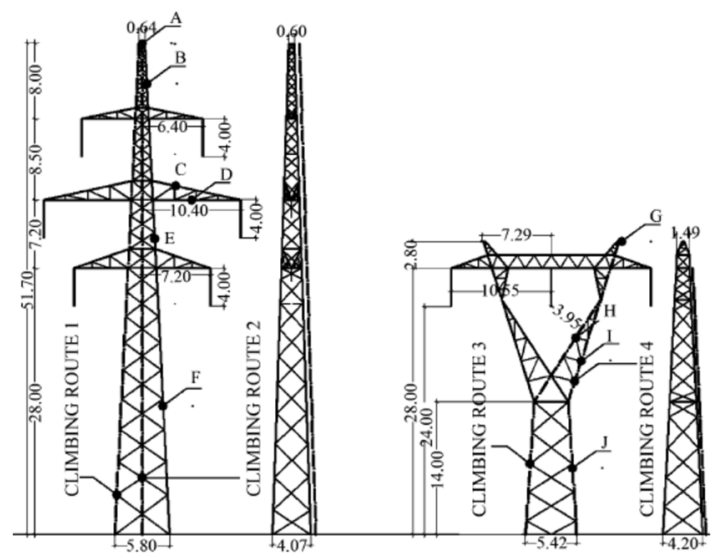

Figure 1. Different climbing routes in tower [13]

A graphical comparison of the Experimental data, fitted curves from the Regressions, the Neural Network, Support Vector Machines and the Hybrid Technique are shown in figure 2 and 3.

Modeling and prediction values for different climbing routes are shown in figure 2 and 3. The four graphs on left hand side (LHS) out of total eight shows performance of neural network, regression, SVM and hybrid technique compared with experiment data for electric field vs height from ground in climbing route 1,2, 3 and 4 . Rest of the four graphs on right hand side (RHS) represents prediction performance for both fields.

For prediction, the height of the tower is increased to $5 \mathrm{~m}$ from the normal height and results are analyzed. From the results it is clear that if we increase the tower height, the electric field will nearly equal to values shown in figure 2 and 3. 



Figure 2. (LHS) Performance of neural network, regression, SVM and hybrid technique compared with experiment data for electric field vs height from ground in climbing routes and (RHS) Prediction of electric field for increase in height using proposed method for climbing routes. 
GA Kulkarni and WZ Gandhare - Acta Technica Jaurinensis, Vol. 10, No. 1, pp. 50-58, 2017
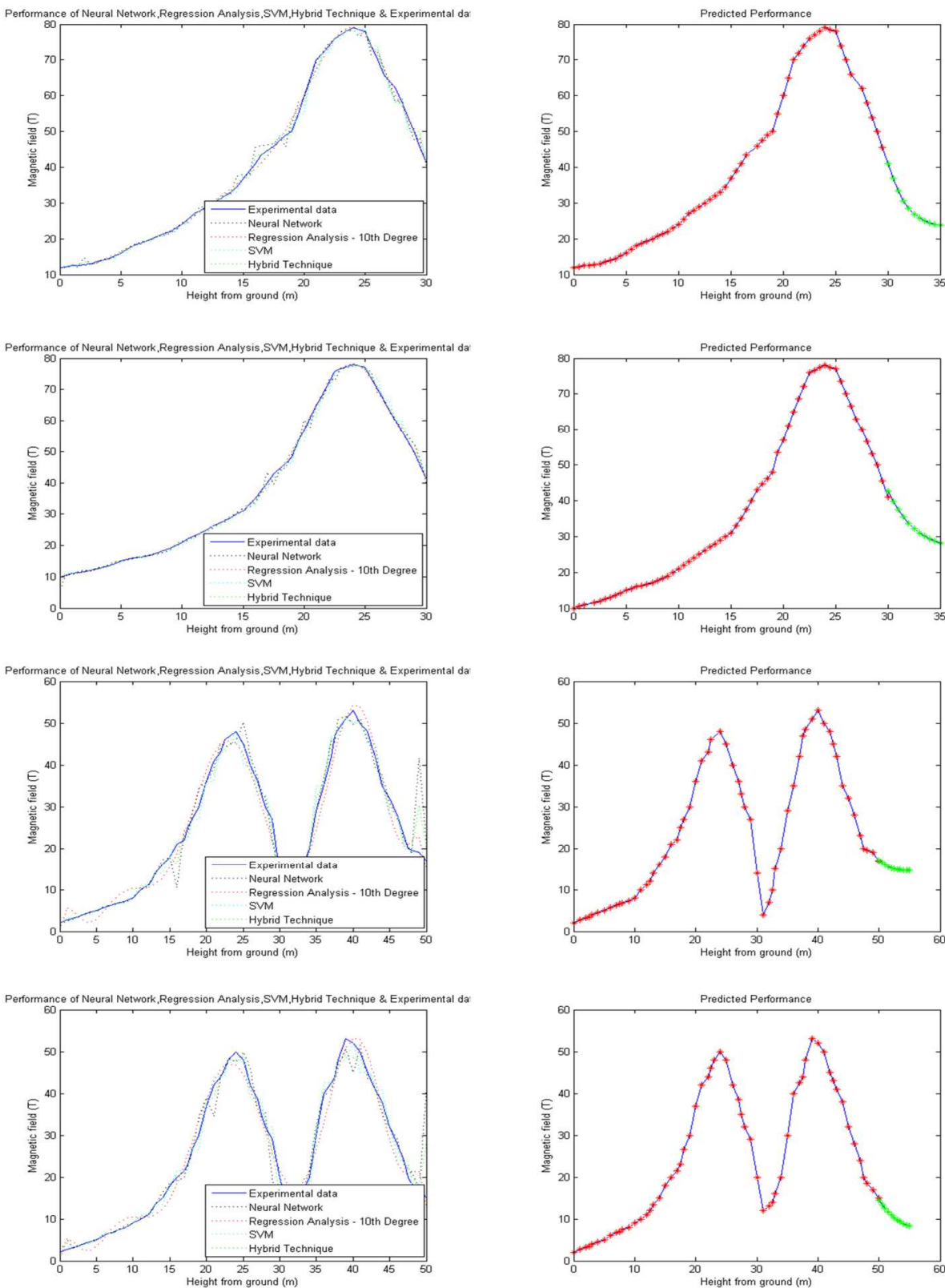

Figure 3. (LHS) Performance of neural network, regression, SVM and hybrid technique compared with experiment data for electric field vs height from ground in climbing routes and (RHS) Prediction of electric field for increase in height using proposed method for climbing routes. 


\section{Conclusions}

Hybrid model to improve predictive performances is proposed. These gives improved results compared to the predictive performances of standalone models $(\mathrm{NN}$, SVM).

The model performance of our hybrid technique was compared with SVM and NN individually. Waveforms show that the hybrid technique is better choice for prediction of EM fields around HVTL.

From the performance result it is clear that proposed method can be efficiently used to determine extensive exposure prone zones to avoid probable detrimental effects due to electromagnetic fields around hotline workers.

\section{References}

[1] Meah K, Ula S: Comparative Evaluation of HVDC and HVAV Transmission Systems. Power Engineering Society General Meeting, IEEE, pp. 1-5, June 2007.

DOI: $10.1109 /$ PES.2007.385993

[2] Kamel NM, Gawad A: An Investigation into Magnetic Field Management under Power Transmission Lines Using Delta Configurations. The Open Environmental Engineering Journal, Vol. 2, pp. 50-67, 2009.

DOI: $10.2174 / 1874829500902010050$

[3] Bonnell JA: Effects of Electric Fields near Power-Transmission Plant. Journal of the Royal Society of Medicine, Vol. 75, No. 12, pp. 933-941, Dec 1982.

[4] Maalej NM, Belhadj CA, Abdel-Galil TK, Habiballah IB: Visible Human Utilization to Render Induced Electric Field and Current Density Images Inside the Human. Proceedings of the IEEE, Vol. 97, No. 12, pp. 2053-2059, Dec 2009.

DOI: $10.1109 /$ JPROC. 2009.2031668

[5] Kulkarni GA, Gandhare WZ: Modeling of Electric and Magnetic Fields around 132kv Transmission Line. Acta Technica Jaurinensis, Vol. 7, No. 3, pp. 247257, 2014.

DOI: 10.14513/actatechjaur.v7.n3.302

[6] Maalej NM, Belhadj CA: External and Internal Electromagnetic Exposures of Workers near High Voltage Power Lines. Progress in Electromagnetic Research C, Vol. 19, pp. 191-205, 2011.

DOI: 10.2528/PIERC10110601

[7] Alkoot FM, Zaeri N: Measurement of Low Frequency Electromagnetic Radiation Emitted From Overhead Power Lines in the State of Kuwait. Proceedings of the $7^{\text {th }}$ WSEAS International Conference on Power Systems, Beijing, China, pp. 186-191, September 2007.

[8] Xu L, Krzyzak A: Methods of Combining Multiple Classifiers and Their Applications to Handwriting Recognition. IEEE Transactions on Systems, Man and Cybernetics, Vol. 22, No. 3, pp. 418-435, 1992.

DOI: $\underline{10.1109 / 21.155943}$ 
[9] Razi MA, Athappilly K: A Comparative Predictive Analysis of Neural Networks (NNs), Nonlinear Regression and Classification and Regression Tree (CART) Models. Expert Systems with Applications, Vol. 29, No. 1, pp. 65-74, 2005.

DOI: $10.1016 /$ j.eswa.2005.01.006

[10] Balasubramanyam C, Ajay MS, Spandana KR, Shetty AB, Seetharamu KN: Curve Fitting For Coarse Data Using Artificial Neural Network. WSEAS Transaction on Mathematics, Vol. 13, pp. 406-415, 2014.

[11] Abuthakeer SS, Mohanram PV, Kumar GM: Prediction and Control of Cutting Tool Vibration in CNC Lathe With Anova and ANN. International Journal of Lean Thinking, Vol. 2, No. 1, pp. 1-23, June 2011.

[12] Woodsend K, Gondzio J: Hybrid MPI/Open MP Parallel Linear Support Vector Machine Training: Journal of Machine Learning Research, Vol. 10, pp. 1937-1953, 2009.

[13]Zemljaric B: Calculation of the Connected Magnetic and Electric Fields around an Overhead-Line Tower for an Estimation of their Influence on Maintnance Personnel. IEEE transaction on power delivery, Vol. 26, No. 1, pp. 467-474, January 2011.

DOI: 10.1109/TPWRD.2010.2064342

[14] Glenn Lahodny Jr., Curve Fitting and Parameter Estimation, Spring 2015, http://www.math.tamu.edu/ glahodny/Math442/Curve\%20Fitting.pdf 